\title{
Pressure and flow properties of cannulae for extracorporeal membrane oxygenation I: return (arterial) cannulae
}

\author{
Lars Mikael Broman, ',2,3 (D) Lisa Prahl Wittberg, ${ }^{4}$ \\ C Jerker Westlund,' Martijn Gilbers, ${ }^{5,6}$ Luisa Perry da Câmara,7 \\ Justyna Swol, 3,8 (D) Fabio S Taccone, ${ }^{3,9}$ Maximilian V Malfertheiner, 3,10 \\ Matteo Di Nardo, 3,11 (iD Leen Vercaemst, 3,12 Nicholas A Barrett, ${ }^{3,13}$ \\ Federico Pappalardo, 3,14 Jan Belohlavek, 3,15 Thomas Müller, 3,10 \\ Mirko Belliato ${ }^{3,16}$ and Roberto Lorusso ${ }^{3,5}$
}

\begin{abstract}
Adequate extracorporeal membrane oxygenation support in the adult requires cannulae permitting blood flows up to 6-8 L/minute. In accordance with Poiseuille's law, flow is proportional to the fourth power of cannula inner diameter and inversely proportional to its length. Poiseuille's law can be applied to obtain the pressure drop of an incompressible, Newtonian fluid (such as water) flowing in a cylindrical tube. However, as blood is a pseudoplastic non-Newtonian fluid, the validity of Poiseuille's law is questionable for prediction of cannula properties in clinical practice. Pressure-flow charts with non-Newtonian fluids, such as blood, are typically not provided by the manufacturers. A standardized laboratory test of return (arterial) cannulae for extracorporeal membrane oxygenation was performed. The aim was to determine pressure-flow data with human whole blood in addition to manufacturers' water tests to facilitate an appropriate choice of cannula for the desired flow range. In total, 14 cannulae from three manufacturers were tested. Data concerning design, characteristics, and performance were graphically presented for each tested cannula. Measured blood flows were in most cases $3-21 \%$ lower than those provided by manufacturers. This was most pronounced in the narrow cannulae $(15-17 \mathrm{Fr})$ where the reduction ranged from $27 \%$ to $40 \%$ at low flows and $5-15 \%$ in the upper flow range. These differences were less apparent with increasing cannula diameter. There was a marked disparity between manufacturers. Based on the measured results, testing of cannulae including whole blood flows in a standardized bench test would be recommended.
\end{abstract}

\section{Keywords}

arterial; return; extracorporeal membrane oxygenation; cannula; pressure flow; blood; water; extracorporeal life support

'ECMO Center Karolinska, Department of Pediatric Perioperative Medicine and Intensive Care, Karolinska University Hospital, Stockholm, Sweden

2Department of Physiology and Pharmacology, Karolinska Institutet, Stockholm, Sweden

${ }^{3}$ Working Group on Innovation and Technology, EuroElso, Newcastle upon Tyne, UK

${ }^{4}$ The Linné Flow Centre and BioMEx Centre, Department of Mechanics, KTH Royal Institute of Technology, Stockholm, Sweden ${ }^{5}$ Department of Cardio-Thoracic Surgery, Heart \& Vascular Centre, Cardiovascular Research Institute Maastricht (CARIM), Maastricht University Medical Hospital, Maastricht, The Netherlands ${ }^{6}$ Department of Physiology, Maastricht University, Maastricht, The Netherlands

${ }^{7}$ Centro Hospitalar de Lisboa Central, Hospital Curry Cabral, Lisbon, Portugal

${ }^{8}$ Department of Pulmonology, Intensive Care Medicine, Paracelsus Medical University, Nuremberg, Germany

${ }^{9}$ Department of Intensive Care, Hôpital Erasme, Université Libre de Bruxelles (ULB), Brussels, Belgium
${ }^{10}$ Department of Internal Medicine II, Cardiology and Pneumology, University Medical Center Regensburg, Regensburg, Germany

I Pediatric Intensive Care Unit, Children's Hospital Bambino Gesù, IRCCS, Rome, Italy

${ }^{12}$ Department of Perfusion, University Hospital Gasthuisberg, Leuven, Belgium ${ }^{13}$ Department of Critical Care and Severe Respiratory Failure Service, Guy's and St Thomas' NHS Foundation Trust, London, UK

${ }^{14}$ Advanced Heart Failure and Mechanical Circulatory Support Program, San Raffaele Hospital, Vita-Salute San Raffaele University, Milan, Italy

${ }^{15}$ 2nd Department of Medicine-Department of Cardiovascular Medicine, General University Hospital in Prague and First Faculty of Medicine, Charles University in Prague, Prague, Czech Republic 16U.O.C. Anestesia e Rianimazione I, Fondazione IRCCS Policlinico San Matteo, Pavia, Italy

Corresponding author:

Lars Mikael Broman, ECMO Center Karolinska, Department of Pediatric Perioperative Medicine and Intensive Care, Karolinska University Hospital, Eugeniavagen 23, I7I76 Stockholm, Sweden. Email: lars.broman@sll.se 


\section{Introduction}

Adequate vascular access is essential for the performance of extracorporeal membrane oxygenation (ECMO). Cannulae for access are manufactured and supplied by several companies. The specifications for the interaction of cannulae on inflammation and coagulation are regulated by International Standard (ISO 10993-4). However, there are no recommendations provided as to the ideal pressure-flow relation in respect to prolonged cannula use to prevent blood cell damage, turbulent flows, jets imposing increased stress levels onto the blood vessels, air bubble generation in negative pressure settings, and so on. Likewise, there is no standardized testing procedure for manufacturers concerning ECMO cannula flow performance. Paulsen et al. ${ }^{1}$ tested a large number of cannulae using glycerol solution. Most manufacturers present pressure-flow charts of their own using room temperature $\left(20-24^{\circ} \mathrm{C}\right)$ and water as the medium. From clinical experience and discussions with researchers in fluid mechanics/engineering, these measurements cannot be directly extrapolated to human use because of the flow characteristics of blood as a non-Newtonian fluid. The volume flow through a cylindrical tube for Newtonian fluids (i.e. water, saline, glycerol solution, etc.) is obtained from Poiseuille's law (equation (1)) for Reynolds number within the laminar range. From Poiseuille's law, the volume flow is proportional to the fourth power of the radius. Hence, a larger diameter cannula achieves far greater flow. The pressure-drop $\left(\mathrm{P}_{1}-\mathrm{P}_{2}\right)$ over the cannula is proportional to volume flow, whereas viscosity inversely related to flow

$$
\mathrm{Q}=\frac{\mathrm{r}^{4} \times \pi \times\left(\mathrm{P}_{1}-\mathrm{P}_{2}\right)}{8 \times \mathrm{L} \times \eta}
$$

where "Q" is the volume flow, "r" radius, "(P1-P2)" is the pressure-drop, " $L$ " is the length of the cylinder, and " $\eta$ " is the viscosity. Note that blood is a non-Newtonian pseudoplastic fluid exhibiting shear thinning that with increasing shear stress displays a decrease in viscosity. ${ }^{2,3}$ Moreover, at shear rates greater than approximately $100 /$ second, blood displays a Newtonian viscosity behavior. ${ }^{4-7}$

Cannulae as well as the speed/rotation of the centrifugal pump may cause red blood cell (RBC) damage and platelet activation if subject to high shear stress for a certain amount of time. Hemolysis and, hence, plasma-free hemoglobin $(\mathrm{Hg})$ scavenges all nitric oxide $(\mathrm{NO})$ in the microcirculation which promotes vasoconstriction, increased inflammation, and coagulation. ${ }^{7-10}$ Shear stresses found in the flow are influenced by the geometry such as the internal radius, length of the cannula, as well as the flow regime (laminar, transitional, or turbulent), that in turn will affect the pressure drop over the cannula. ${ }^{11}$ The choice of return cannula is especially important in prolonged use, since high shear stress may

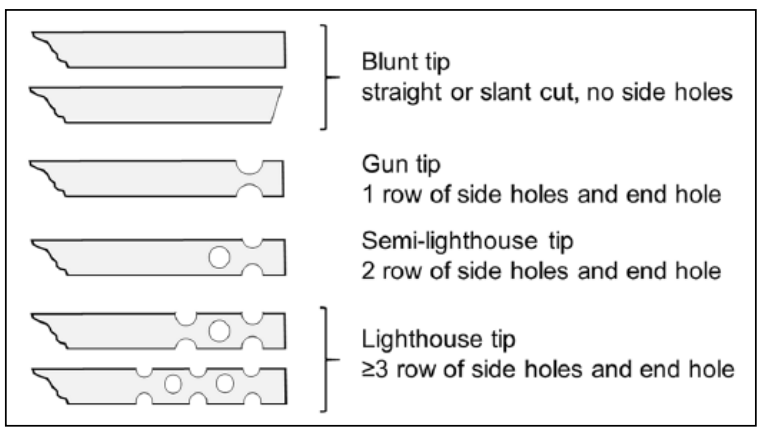

Figure I. Figure I displays contemporary single-staged tip designs for cannulae used in extracorporeal membrane oxygenation (ECMO). For return (arterial) cannulae all these designs are used with variations of rows of holes (as shown), sizes, diameters, and so on.

activate platelets, ${ }^{12,13}$ affect Von Willebrand factor, ${ }^{14}$ and, as mentioned, increase $\mathrm{RBC}$ damage affecting both $\mathrm{NO}$ in the microcirculation as well as the rheological properties of RBCs. ${ }^{15}$

For any cannula, there is a fluid flow limit beyond which laminar flow cannot be maintained; beyond this point, the flow becomes unsteady and transitional. Further increasing the flow rate leads to a turbulent flow. At identical shear stress, turbulent flow produces far more blood trauma than laminar flow. ${ }^{16}$ Although blood flow is expected to transition to turbulent at larger flow velocities than water, it is important to assess this difference as well as provide access to hydrodynamic flow charts from in vitro testing using blood rather than water.

The aim of this study was to test cannulae using human whole blood (HWB) to define pressure-flow charts for some commonly used return cannulae used during ECMO for peripheral cannulation. Performance comparison between cannulae did not fall within the agreement of the study. The importance of the results lies in the possibility to provide a better guidance regarding the choice of cannula size for required volume flow to the patient and thus decrease the risk of complications associated with turbulent flow.

\section{Material and methods}

Manufacturers were approached to support the laboratory tests with cannulae marketed for ECMO. No financial support was asked for or accepted. All cannulae were coded and blinded to investigators during test. For compilation of test data, each cannula's identification was revealed and double checked against code key and test chart.

Arterial or venous return cannulae for peripheral cannulation are in general single staged and shorter than the venous drainage cannulae. The most common tip designs are shown in Figure 1. Cannula features, including manual caliper measurements of the tested specimens, are displayed in Table 1. The cannula size is given in French 
Table I. Table I shows independent caliper measurements performed by the authors and other characteristics for return (arterial) cannulae used for extracorporeal membrane oxygenation.

\begin{tabular}{|c|c|c|c|c|c|c|c|c|c|c|}
\hline Manufacturer & Type & Model & $\begin{array}{l}\text { Size } \\
\text { (Fr) }\end{array}$ & $\begin{array}{l}\text { Maximum } \\
\text { insertion } \\
\text { length } \\
(\mathrm{mm})\end{array}$ & $\begin{array}{l}\text { Flow at } \Delta \mathrm{P} \\
80 \mathrm{mmHg} \\
(\mathrm{mL} / \mathrm{min}- \\
\text { ute })\end{array}$ & $\begin{array}{l}\text { Tip } \\
\text { design }\end{array}$ & $\begin{array}{l}\text { Tip to cent- } \\
\text { er of most } \\
\text { proximal } \\
\text { hole }(\mathrm{mm})\end{array}$ & $\begin{array}{l}\text { Distance } \\
\text { tip to } \\
\text { wire } \\
(\mathrm{mm})\end{array}$ & $\begin{array}{l}\text { Side hole diam- } \\
\text { eter } \times \text { number } \\
\text { of holes per } \\
\text { row } \times \text { rows } \\
(\mathrm{mm} \times \mathrm{n} \times \mathrm{n})\end{array}$ & $\begin{array}{l}\text { Outer } \\
\text { diameter by } \\
\text { caliper (Fr ref. } \\
\mathrm{mm} \text { ) }\end{array}$ \\
\hline \multirow[t]{7}{*}{ Maquet } & HLS & $15 \mathrm{Fr} / 15 \mathrm{~cm}$ & 15 & 146 & 2,480 & Gun & 10 & 1.5 & $2.3 \times 2 \times 1$ & $5.0-5.15(5.0)$ \\
\hline & HLS & $15 \mathrm{Fr} / 23 \mathrm{~cm}$ & 15 & 226 & 2,150 & Gun & 10 & 1.5 & $2.5 \times 2 \times 1$ & $5.1-5.15(5.0)$ \\
\hline & HLS & $17 \mathrm{Fr} / 15 \mathrm{~cm}$ & 17 & 147 & 3,420 & Gun & 10 & 1.5 & $2.3 \times 2 \times 1$ & $5.7-5.9(5.7)$ \\
\hline & HLS & $17 \mathrm{Fr} / 23 \mathrm{~cm}$ & 17 & 227 & 3,000 & Gun & 10 & 1.5 & $2.0 \times 2 \times 1$ & $5.8-6.0(5.7)$ \\
\hline & HLS & $21 \mathrm{Fr} / 15 \mathrm{~cm}$ & 21 & 148 & 5,800 & Gun & 10 & 1.5 & $2.5 \times 2 \times 1$ & 7.I-7.3(7.0) \\
\hline & HLS & $21 \mathrm{Fr} / 23 \mathrm{~cm}$ & 21 & 230 & 5,440 & Gun & 10 & 1.5 & $2.5 \times 2 \times 1$ & $6.85-7.15(7.0)$ \\
\hline & HLS & $23 \mathrm{Fr} / 23 \mathrm{~cm}$ & 23 & 231 & 6,850 & Gun & 10 & 2 & $2.5 \times 2 \times 1$ & 7.8-8.0(7.7) \\
\hline \multirow[t]{3}{*}{ Medos } & MEFKA & MEFKAI6L & 16 & 162 & 2,620 & $\mathrm{LgH}$ & 33 & 37 & $2.0 \times 2 \times 6$ & $5.4-5.45(5.3)$ \\
\hline & MEFKA & MEFKA20L & 20 & 161 & 4,960 & $\mathrm{LgH}$ & 33 & 38 & $2.0 \times 2 \times 6$ & $6.7-6.9(6.7)$ \\
\hline & MEFKA & MEFKA24L & 24 & 167 & 8,040 & $\mathrm{LgH}$ & 34 & 38 & $2.0 \times 2 \times 6$ & $7.85-8.0(8.0)$ \\
\hline Medtronic & NextGen & $15 \mathrm{Fr} / 31.8$ & 15 & 175 & 2,350 & $\mathrm{LgH}$ & 21 & 2 & $2.0 \times 2 \times 3$ & $5.0(5.0)$ \\
\hline \multirow[t]{3}{*}{ Bio-Medicus } & NextGen & $|7 \mathrm{Fr} / 3| .8$ & 17 & 175 & 3,330 & $\mathrm{LgH}$ & 22 & 2 & $2.0 \times 2 \times 3$ & $5.7-5.85(5.7)$ \\
\hline & NextGen & $21 \mathrm{Fr} / 31.8$ & 21 & 177 & 5,870 & $\mathrm{LgH}$ & 22 & 2.3 & $2.5 \times 2 \times 3$ & $6.9-7.0(7.0)$ \\
\hline & NextGen & $23 \mathrm{Fr} / 3 \mathrm{I} .8$ & 23 & 180 & 7,300 & $\mathrm{LgH}$ & 21 & 2.4 & $3.0 \times 2 \times 3$ & 7.5(7.7) \\
\hline
\end{tabular}

Fr: French; LgH: lighthouse tip.

All cannulae carry $3 / 8^{\prime \prime}$ connectors with a Luer lock at the side; outer diameter $(\mathrm{Fr} \times \mathrm{I} / 3 \mathrm{~mm})$.

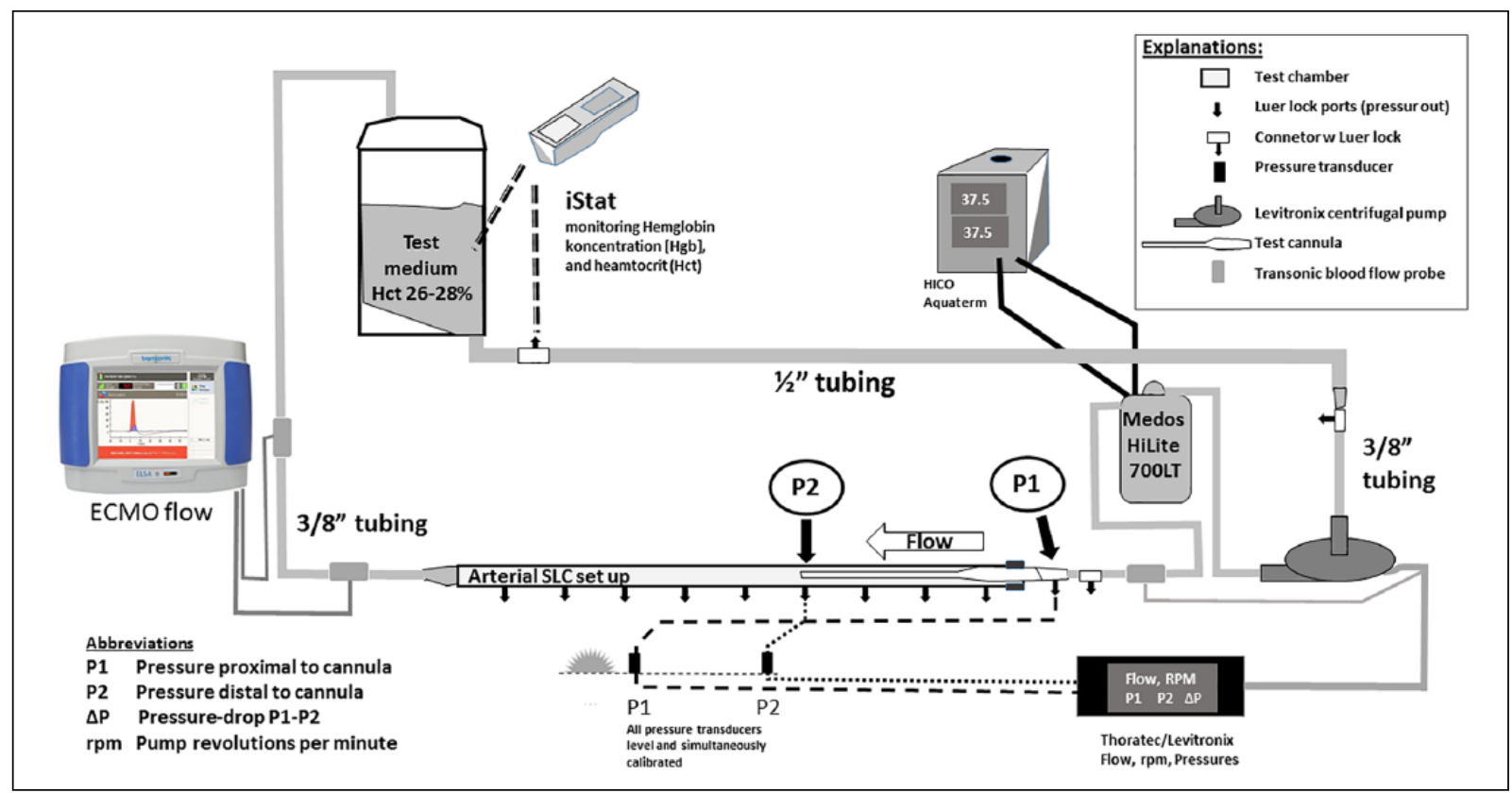

Figure 2. Figure 2 shows the mock loop for flow tests of return (arterial) cannulae used extracorporeal membrane oxygenation (ECMO).

(Fr; $1 \mathrm{Fr}=1 / 3 \mathrm{~mm}$ ). The measurement refers to the outer cannula diameter. Most manufacturers regard the distance from the tip to where the cannula starts to increase in diameter, that is, the maximum insertion length. However, Medtronic (Medtronic International Trading Sarl, Tolochenaz, Switzerland) reports the length from the tip to the $3 / 8^{\prime \prime}$ connector for the Bio-Medicus
NextGen cannulae. In Table 1, the insertion length is showed for all cannulae, and all designs were adequate for a percutaneous approach to cannulation due to the semirigid to rigid tapering transition to the mandrel.

The mock loop was composed of a Thoratec/ Levitronix CentriMag pump (Abbott, Illinois, USA), a reservoir, and $1 / 2^{\prime \prime}$ and $3 / 8^{\prime \prime}$ tubing as default (Figure 2 ). 
For temperature stability and oxygenation of the blood during the tests, a Medos hilite 7000 LT (Xenios, Heilbronn, Germany) membrane lung was used. A blood warmer (HICO-Aquatherm 660, Hirtz \& Co., Cologne, Germany) was used for temperature control set to $37.5^{\circ} \mathrm{C}$. A test chamber was developed from a $750 \mathrm{~mm}, 40 \times 4 \mathrm{~mm}$ Plexiglass ${ }^{\mathrm{TM}}$ tube with Luer lock ports every $71 \mathrm{~mm}$ for pressure monitoring at the position of the cannula tip.

The amount of HWB available for the test was six units (expired and donated blood), corresponding to approximately $2.4 \mathrm{~L}$. The evening before the experiments, a minimalized test circuit was assembled with uncoated tubing $(1,450 \mathrm{~mL}$ plus the reservoir, Figure 2$)$ and primed with saline and $20 \%$ albumin. Afterwards, the circuit was left running with sweep gas overnight for leakage control. At 07:00 AM on the test day, the circuit was blood primed, and a hematocrit (Hct) feasible in ECMO patients of $27 \pm 1 \%$ was maintained over the runs. ${ }^{17,18}$ Oxygen was given via the membrane lung to mimic an "arterial" milieu for the blood pumped through the cannula.

For control of the test medium, Hgb concentration, Hct, and blood samples were analyzed regularly with an iSTAT device (Abbott Point of Care, Maidenhead, UK). No anticoagulative regimen was used (besides what already was in the units delivered). Blood flow and revolutions per minute $(\mathrm{rpm})$ were monitored via the Thoratec, and flow measurements were performed separately using an extracorporeal life support assurance (ELSA) device (Transonic Systems Inc., Ithaca, NY, USA) stand-alone unit for higher flow resolution and accuracy, since the ELSA uses dual flow transducers. Pressures were measured and displayed via the Thoratec.

\section{Pressure measurements and calculations}

For each volume flow, the pressure gradient over the cannula was assessed by measuring the cannula inlet (proximal) pressure $\left(\mathrm{P}_{1}\right)$ at the Luer lock port available on the $3 / 8^{\prime \prime}$ cannula connector. To obtain the distal outlet pressure $\left(\mathrm{P}_{2}\right)$, the cannula was placed such that a Luer lock port was close to the tip/infusion ports (Figure 2). Pressure transducers were placed in level with the horizontal test chamber and zeroed.

Data collected concerning cannula code, date/time, blood temperature, blood flow (L/minute), pump rpm, $\mathrm{P}_{1}$ and $\mathrm{P}_{2}(\mathrm{mmHg})$, blood gases, and Hct were checked. The pressure drop over the test specimen was calculated as $\Delta \mathrm{P}=\mathrm{P}_{1}-\mathrm{P}_{2}$ at any flow. From these assessments, pressure-flow graphs were obtained. Data acquisition was carried out for a single measurement run for each cannula. This was motivated by previous own testing and similar results to those by Wang et al. ${ }^{19}$ that showed limited variability. Moreover, additional pressure measure- ments were carried out for random cannulae to confirm methodology.

To display differences in flow properties between runs made with blood compared to water, the manufacturers' pressure-flow charts made with water were collected from the public domain on the Internet. Pressure-flow data were extracted and added to each respective cannula's pressure-flow graph acquired from this investigation.

\section{Ethical concerns}

Ethical approval was waived according to Swedish law and contact with the Department of Transfusion Medicine, Karolinska University Hospital, Huddinge, Stockholm, who donated the blood otherwise up for destruction due to passing the 42 days (maximum allowed storage time). Thus, no consent was needed from the donors.

\section{Statistics}

Cannulae were not directly compared with each other in accordance with our agreement with the manufacturers. Thus, all results are reported per manufacturer. Consequently, no statistical analysis was undertaken.

\section{Results}

In total, 14 single-lumen cannulae for adult and larger pediatric patients were tested for flow and pressure relationship using whole blood. For a cannula dimension not tested in this study, a flow approximation could be obtained by placing the "missing cannula" in the space between two closest cannulae in sizes tested in the appropriate graph in Figure 3. The manufacturers supporting this study where Maquet (Maquet Cardiopulmonary, Getinge Group, Rastatt, Germany), Medtronic International Trading, and Xenios via Octopus Medical AB/Limedic AB (Täby, Sweden).

In Figure 3, cannulae pressure-flow curves using HWB are shown for each manufacturer's cannulae. The respective insertion lengths and tip designs are shown in Table 1. In terms of overall flow, the tip designs did not show any impact on flow rate, rather flow was dependent on diameter and effective (i.e. insertion) length.

Each cannula's pressure-flow obtained from the current investigation with blood and the manufacturers' water runs merged graphically are presented in Supplemental Digital File 1. To pump the more viscous blood to water, an additional pressure gradient of $20-90 \%$ was needed to achieve the same flow. The BioMedicus cannulae showed a decrease in the difference between blood and water as a function of higher blood 


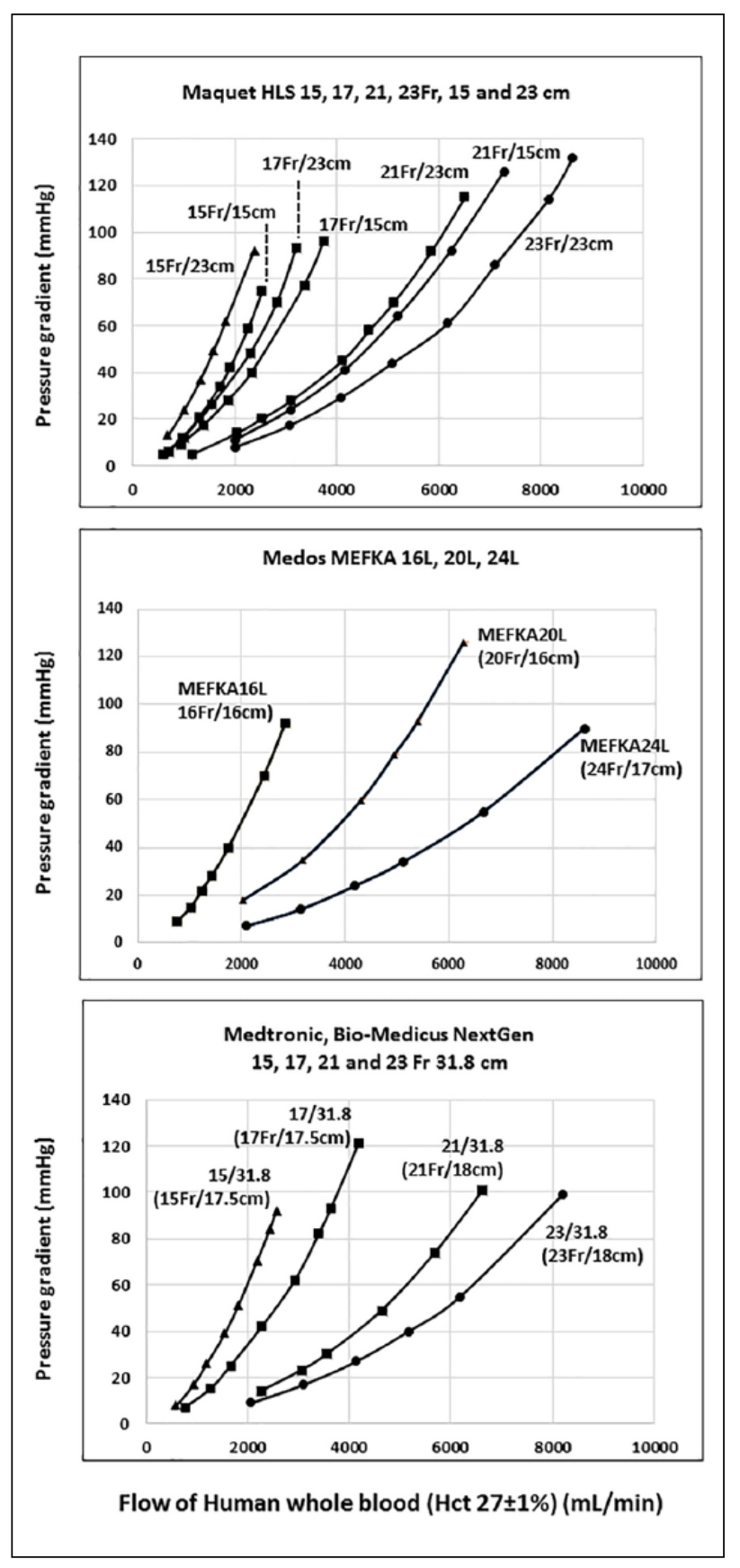

Figure 3. Figure 3 shows pressure-flow relationship for different cannulae sizes from brands referred to in each panel. Fr: French.

Cannula type and size/length according to manufacturer's preference shown at each bar in graph; concerning length, there is no consensus among manufacturers; cannula diameter and maximum insertion length is given in brackets below the name given by the manufacturer, respectively; I $\mathrm{Fr}=1 / 3 \mathrm{~mm}$ (outer cannula diameter).

flows and larger cannula size. The same was observed for the Maquet family except for the $23 \mathrm{Fr}$ cannula which performed similar with blood compared to water in the tested flow range. The Medos cannulae showed some overlap for the $16 \mathrm{Fr}$ cannula with a further increase in flow improvement for blood flow compared to water with increased flow and size.

\section{Discussion}

In total, 14 cannulae marketed for ECMO were tested. Return cannulae for adult peripheral cannulation are often shorter than the venous cannulae. In this context, it should be mentioned that several manufacturers market the same device as return (arterial) or drainage (venous), with the only difference being a Luer lock adapter on the side of the $3 / 8^{\prime \prime}$ connector for the return cannula. For the adults and children above 6-10 years of age (ca $20-40 \mathrm{~kg}$ ), the most common site for reinfusion of oxygenated blood for venoarterial ECMO is via cannulation of a femoral artery (cannula tip in iliac artery). A distal perfusion cannula is advocated to ensure adequate blood flow to the leg, and this may be connected to the reinfusion cannula via the Luer lock port. ${ }^{16}$ For infusion during venovenous ECMO, this return cannula is mostly inserted via the internal jugular vein, but configurations via a femoral or subclavian vein also exist. ${ }^{20}$

The results of this study compared with the manufacturer's flow charts, published in the public domain, showed that the pressure gradient needed for a given volume flow in most cases was lower using water compared with HWB. However, the fractional additional pressure needed to increase blood flow decreased with increasing flow, in accordance with blood being a shear thinning fluid. In the investigation by Andersen et al., ${ }^{21}$ a higher degree of turbulent flow was found for a given flow in cannulae for cardiopulmonary bypass with same outer diameter but smaller inner diameter, that is, the effective diameter. This is expected because, for a given flow, a decrease in diameter will directly increase the flow velocity within the cannula and consequently turbulent flow may develop. In another study with a different aim, Wang et al. ${ }^{19}$ compared cannulae of the same diameter and similar length from two different manufacturers pumping human erythrocytes in Ringers lactate (Hct 40\%). The exact model and lengths were not disclosed, but it seems as the better cannula in that study did not outperform the competitor in the current investigation. They also showed that cannulae with less diameter had higher resistance and that different cannulae of similar size differed in flow properties. That any cannula should perform better with blood than water conflicts with accepted physical laws, yet, this was apparently the case in four comparisons, emphasizing the need for standardized methods for cannula performance testing. With uncertain methods for testing, comparisons between cannulae brands, even within similar size and design, based on the manufacturers' individual tests using water cannot be made. Several factors, that is, confounders, may influence test results: mock loop design per se; methodology 
in how the test runs are performed, for example, water bath or vessel equivalent; pressure measuring points; and properties of the fluid used to test flow. In this investigation, cannulae were tested in a random blinded fashion, and the changes between cannula diameters within the same cannula family were consistent across the experiment and with predictions. When the respective manufacturer's water curves were added to the blood flow curves in Supplemental Digital File 1, the starting point along the y-axis had migrated with lower flows demonstrated for blood than water.

On analyzing the pressure-flow (and flow-pressure) curves (Supplemental Digital File 1), obtained with blood, they are congruent with Poiseuille's law (equation (1)) and work by others: ${ }^{4,5,19}$ for return cannulae of smaller size, the pressure difference needed to accomplish a given flow was higher than in the larger cannula specimen of same cannula family. To exemplify, increasing size from 15-17 Fr (same length) increased blood flow by $40-60 \%$ in the pressure gradient range $10-40 \mathrm{mmHg}$. This should not be disregarded in the choice of access devices, especially in low-flow support such as extracorporeal carbon dioxide removal. Chronic hypoxemic patients are also prone to have a high $\mathrm{Hct}$ (i.e. viscosity) which may exacerbate pressure problems in the small diameter cannula. These findings further emphasize the importance of pressure-flow assessments with blood for reliable clinical application and during the cannula design phase.

No apparent difference was observed between the different designs in terms of overall flow, rather the main impact on flow rate was length and diameter. The most common tip designs carry one or several rows of side holes dispersing pressure and flow in contrast to single end-hole design used in small children which may risk intima injury from the flow-jet. One ex-vivo investigation from 1990 examined performance of neonatal cannulae with blunt-tip design being deemed appropriate for carotid placement. ${ }^{22}$ These cannulae (6-14 Fr) would have added value to the study. Unfortunately, no study cannulae for the smaller children were made available by manufacturers.

The strength of this study was that cannulae were tested during constant conditions and a stable Hct. Furthermore, the pressure-drop across the cannula could be measured at multiple points optimizing for a true gradient over the cannula length. Limitations of this study were that no tests with water for replication of manufacturer's data were conducted and the restraints for comparative analysis between different brands of the study. A further limitation of this study was that tests were only able to be performed at one Hct, limiting the interpretation of the pressure-drop in relation to different Hct values. This is an important factor due to the known changes in flow due to the effect of shear thinning. ${ }^{5,6}$ It is also possible that the flow characteristics of HWB which was over 42 days is different to fresh blood in vivo. Similarly, as HWB was used, it is not possible to make a recommendation as to whether fresh animal blood will provide adequate information for clinicians. However, given that the European Union Medical Device Regulation (MDR EU 2017/745) will be fully implemented in May 2020, ${ }^{23}$ this may have the effect of restricting medical devices on the market to those which have received regulatory approval during the implementation period (2020-2024).

Our results suggest that information about cannula characteristics in clinical practice is clearly enhanced by testing both with water and blood. Therefore, in future, this method should be considered to become part of the approval procedure for cannula.

\section{Conclusion}

Single-lumen return cannulae for peripheral ECMO tested using whole human blood showed different flow rates as compared to those reported by manufacturers using water. There is currently no standardized benchtesting regimen for manufacturers to comply with. To allow clinicians to make decisions about which cannula to choose for individual patients, testing of cannulae, including whole blood flow in a standardized bench test, would be recommended.

\section{Acknowledgements}

The authors are grateful to Jan Westin, Department of Medical Technology, Karolinska University Hospital, Stockholm, Sweden, who constructed the test chamber and to the manufacturers that supported this survey (alphabetical order): Getinge, Maquet Cardiopulmonary GmbH, Rastatt, Germany; Medtronic International Trading Sarl, Tolochenaz, Switzerland; Octopus Medical AB/Limedic AB, Täby, Sweden; and Xenios AG, Heilbronn, Germany. L.M.B. designed the concept, test chamber, and mock loop; collected, compiled, and analyzed the data; and drafted the manuscript. R.L. co-designed the concept. C.J.W., M.G., L.P.d.C., and J.S. collected the data. L.M.B., L.P.d.C., R.L., C.J.W., M.G., J.S., J.W., L.P.W., F.S.B., M.V.M., M.D.N., L.V., N.A.B, F.P., J.B., T.M., and M.B. interpreted the data and critically revised the manuscript. Approval for submission was given by all the authors.

\section{Declaration of Conflicting Interests}

The author(s) declared the following potential conflicts of interest with respect to the research, authorship, and/or publication of this article: L.M.B., F.S.B., M.V.M., M.D.N., F.P., and M.B. are on the Medical Advisory Board of Eurosets Srl, Medolla, Modena, Italy. The remaining authors had no conflict of interest to disclose. 


\section{Funding}

The author(s) received no financial support for the research, authorship, and/or publication of this article.

\section{Supplemental material}

Supplemental material for this article is available online.

\section{ORCID iDs}

LarsMikaelBroman (iD https://orcid.org/0000-0003-4124-4581 Lisa PrahlWittberg iD https://orcid.org/0000-0001-9976-8316 Justyna Swol (iD https://orcid.org/0000-0002-2903-092X Matteo Di Nardo (iD https://orcid.org/0000-0003-0051-8080 Roberto Lorusso iD https://orcid.org/0000-0002-1777-2045

\section{References}

1. Paulsen MJ, Orizondo R, Le D, et al. A simple, standard method to characterize pressure/flow performance of vascular access cannulas. ASAIO J 2013; 59: 24-29.

2. Tazraei P, Riasi A, Takabi B. The influence of the nonNewtonian properties of blood on blood-hammer through the posterior cerebral artery. Math Biosci 2015; 264: 119-127.

3. Gijsen FJH, Allanic E, van de Vosse FN, et al. The influence of the non-Newtonian properties of blood on the flow in large arteries: unsteady flow in a $90^{\circ}$ curved tube. J Biomech 1999; 32: 705-713.

4. Kohler K, Valchanov K, Nias G, et al. ECMO cannula review. Perfusion 2013; 28: 114-124.

5. Pries AR, Neuhaus D, Gaehtgens P. Blood viscosity in tube flow: dependence on diameter and hematocrit. Am J Physiol 1992; 263(6 Pt 2): H1770-H1178.

6. Wells RE, Merrill EW, Gabelnick H. Shear-rate dependence of viscosity of blood: interaction of red cells and plasma proteins. Trans Soc Rheol 1962; 7: 9-24.

7. Ulatowski JA, Nishikawa T, Matheson-Urbaitis B, et al. Regional blood flow alterations after bovine fumaryl beta beta-crosslinked hemoglobin transfusion and nitric oxide synthase inhibition. Crit Care Med 1996; 24: 558565.

8. Vogel WM, Dennis RC, Cassidy G, et al. Coronary constrictor effect of stroma-free hemoglobin solutions. Am J Physiol 1986; 251(2 Pt 2): H413-H120.

9. Liu X, Miller MJ, Joshi MS, et al. Diffusion-limited reaction of free nitric oxide with erythrocytes. J Biol Chem 1998; 273: 18709-18713.
10. Vaughn MW, Kuo L, Liao JC. Effective diffusion distance of nitric oxide in the microcirculation. Am J Physiol 1998; 274(5 Pt 2): H1705-H1114.

11. Kameneva M, Burgreen G, Kono K, et al. Effects of turbulent stresses upon mechanical hemolysis: experimental and computational analysis. ASAIO J 2004; 50: 418-423.

12. Cheung PY, Sawicki G, Salas E, et al. The mechanisms of platelet dysfunction during extracorporeal membrane oxygenation in critically ill neonates. Crit Care Med 2000; 28: 2584-2590.

13. Fuchs G, Berg N, Broman LM, et al. Flow-induced platelet activation in components of the extracorporeal membrane oxygenation circuit. Sci Rep 2018; 8: 13985.

14. Kalbhenn J, Schmidt R, Nakamura L, et al. Early diagnosis of acquired von Willebrand Syndrome (AVWS) is elementary for clinical practice in patients treated with ECMO therapy. J Atheroscler Thromb 2015; 22: 265-271.

15. Vercaemst L. Hemolysis in cardiac surgery patients undergoing cardiopulmonary bypass: a review in search of a treatment algorithm. J Extra Corpor Technol 2008; 40: 257-267.

16. Makdisi G, Makdisi T, Wang IW. Use of distal perfusion in peripheral extracorporeal membrane oxygenation. Ann Transl Med 2017; 5: 103.

17. Agerstrand CL, Burkart KM, Abrams DC, et al. Blood conservation in extracorporeal membrane oxygenation for acute respiratory distress syndrome. Ann Thorac Surg 2015; 99: 590-595.

18. Voelker MT, Busch T, Bercker S, et al. Restrictive transfusion practice during extracorporeal membrane oxygenation therapy for severe acute respiratory distress syndrome. Artif Organs 2015; 39: 374-378.

19. Wang S, Force M, Kunselman AR, et al. Hemodynamic evaluation of Avalon Elite bi-caval dual lumen cannulas and femoral arterial cannulas. Artif Organs. Epub ahead of print 1 October 2018. DOI: 10.1111/aor.13318.

20. Lindholm JA. Cannulation for veno-venous extracorporeal membrane oxygenation. J Thorac Dis 2018; 10(Suppl. 5): S606-S612.

21. Andersen MN, Ringgaard S, Hasenkam JM, et al. Quantitative haemodynamic evaluation of aortic cannulas. Perfusion 2004; 19: 323-330.

22. Ehrén H, Frenckner B, Palmér K. In-vitro evaluation of neonatal ECMO cannulae with regard to flow characteristics. Perfusion 1990; 5: 45-51.

23. Di Nardo M, Vercaemst L, Swol J, et al. A narrative review of the technical standards for extracorporeal life support devices (pumps and oxygenators) in Europe. Perfusion 2018; 33: 553-561. 